\title{
PA-1-STK Ovarian Carcinoma Vaccine
}

National Cancer Institute

\section{Source}

National Cancer Institute. PA-1-STK Ovarian Carcinoma Vaccine. NCI Thesaurus. Code C2428.

A cell-based cancer vaccine with potential antineoplastic activity. PA-1-STK ovarian carcinoma vaccine is produced by transducing the ovarian cancer cell line, PA-1, with the herpes simplex thymidine kinase (HSV-tk) gene, resulting in a cell line, PA-1-STK, that permanently expresses the HSV tk gene. Upon transfection into malignant cells, this vaccine is capable of sensitizing tumor cells in response to an antiviral drug such as ganciclovir, which is readily phosphorylated by the TK enzyme to its active form. Administration of ganciclovir following PA-1 STK transfection results in enhanced cytotoxicity of the transfected tumor cells. Additionally, adjacent non-transfected cells are also killed by the activated antiviral drug, a phenomenon referred to as the bystander effect that occurs with this type of suicide-gene transfer technique. 